\title{
Risk of Becoming Cocaine Dependent: Epidemiological Estimates for the United States, 2000-200I
}

\author{
Megan S O'Brien' and James C Anthony*,2 \\ 'Department of Mental Health, Johns Hopkins University Bloomberg School of Public Health, Baltimore, MD, USA; ${ }^{2}$ Department of \\ Epidemiology, Michigan State University College of Human Medicine, East Lansing, MI, USA
}

\begin{abstract}
In this paper, we present new estimates for the risk of becoming cocaine dependent within 24 months after first use of the drug, and study subgroup variation in this risk. The study estimates are based on the National Household Survey on Drug Abuse conducted during 2000-200 I, with a representative sample of US residents aged 12 years and older $(n=1 \mid 4241)$. A total of I08I respondents were found to have used cocaine for the first time within 24 months prior to assessment. Between 5 and $6 \%$ of these recent-onset users had become cocaine dependent since onset of use. Excess risk of recent cocaine dependence soon after onset of cocaine use was found for female subjects, young adults aged 2I-25 years, and non-Hispanic Black/African-Americans. Use of crack-cocaine and taking cocaine by injection were associated with having become cocaine dependent soon after onset of use. These epidemiologic findings help to quantify the continuing public health burden associated with new onsets of cocaine use in the 21 st century. Neuropsychopharmacology (2005) 30, 1006-1018, advance online publication, 23 March 2005; doi: I0.1038/sj.npp. I30068 I
\end{abstract}

Keywords: cocaine; dependence; logistic regression; epidemiology; recent-onset

\section{INTRODUCTION}

In this paper, the main aims are to improve estimates for the risk of becoming cocaine dependent in the first months and years after onset of cocaine use, and to study suspected determinants of becoming dependent within 24 months after cocaine use starts. As background to this research on the incidence of cocaine dependence, we begin with some recently published estimates of the prevalence of recent cocaine use in the US. To illustrate, during calendar year (CY) 2001, an estimated 5.3-6.3 million community-dwelling residents of the US consumed cocaine on at least one occasion. This range from 5.3 to 6.3 million reflects a $95 \%$ confidence interval based on the CY2002 National Household Survey on Drug Abuse (NHSDA). The corresponding point estimate is 5.9 million users, or $2.6 \%$ of the study population. Among the estimated 5.9 million recently active cocaine users, roughly 1.2 million are recent-onset incident users who started their cocaine use in CY2001 (SAMHSA, 2003). That is, one out of every five active users in CY2001 were first-time users.

\footnotetext{
*Correspondence: Dr JC Anthony, Department of Epidemiology, Michigan State University College of Human Medicine, B60I West Fee Hall, East Lansing, Michigan, USA, Tel: + I 517353 8623, Fax: + I 517 432 I I30, E-mail: janthony@msu.edu

Received 5 November 2003; revised 6 October 2004; accepted 15 December 2004

Online publication: 5 January 2005 at http://www.acnp.org/citations/ NPPO I05050305 I 6/default.pdf
}

As described by Wagner and Anthony (2002), this ratio is determined, in part, by the time-dynamics of the cocaine dependence process and a rapid onset of dependence soon after the start of cocaine use, which is one reason why so many individuals are found to have started cocaine use in prior years but continue to use cocaine in recent intervals. According to estimates from the National Comorbidity Surveys of the early 1990s (NCS), when the NCS study sample has been restricted to individuals who have just started to use cocaine, the estimated risk of becoming a case of cocaine dependence is about $5 \%$ per year for the first $1-2$ years after first use. Thereafter, the year-by-year risk declines for each year that passes from the year of first use. This pattern represents a more 'explosive' onset of cocaine dependence after onset of cocaine use, as compared to the more insidious or languorous onset seen for cannabis dependence and for alcohol dependence after first use of these drugs (Wagner and Anthony, 2002).

Whereas we started this work hoping to replicate our own research group's prior findings on the explosive risk of becoming a case of cocaine dependence in the 1-2 years after first use of cocaine, there are two important reasons why we might obtain different estimates. First, these new estimates are based upon the more recently released CY2000 and CY2001 NHSDA public use data sets. As such, we are now at a different stage in the cocaine epidemic that peaked in the 1980s, and some might argue that the cocaine epidemic is over or that annual incidence rates have reached endemic plateau values (eg see Anthony, 1992; Behrens et al, 1999). Second, DSM-IV diagnostic criteria for cocaine 
dependence and the measurement approach of the NHSDA differ somewhat from the DSM-IIIR criteria and methods used in the NCS 10 years ago (Diagnostic and Statistical Manual of Mental Disorders, DSM; APA, 1987, 1994). In addition, the NCS sample estimates are based on any respondent with a lifetime history of cocaine use, and some were asked to report about cocaine dependence problems experienced many years before the NCS assessment in the early 1990s. In contrast, these new NHSDA estimates all are based on recent-onset cocaine users identified since January 2000. For most of these recent-onset users, the maximum interval of recall of cocaine-dependence problems is measured in 0-12 months, some in 1-2 years, none in decades. Moreover, focus on the most recent onset cocaine users may help to constrain problems of differential attrition that can occur in cross-sectional samples of the population. We return to this issue in the discussion of this paper's new study estimates on risk of becoming cocaine dependent once cocaine use has started.

Some readers may have seen cocaine dependence prevalence estimates reported by other research groups, including estimates for the proportion of recently-active cocaine users who are recent cases of cocaine dependence (see Chen and Kandel, 2002; SAMHSA, 2002a, 2003). For example, based on CY2002 estimates, roughly 15-19\% of recently active cocaine users are cocaine dependent (SAMHSA, 2003). This new report is different. Its focus is specifically upon the risk of becoming cocaine dependent within the span of the first 24 months after onset of cocaine use. We wish to check whether the recent evidence is consistent with the explosive risk of becoming cocaine dependent in the first years and months after onset of cocaine use as was disclosed in the NCS estimates cited above, and to learn which subgroups of recent-onset cocaine users are becoming cocaine dependent soon after onset of cocaine use $v s$ those who have not developed the cocaine-dependence syndrome as defined by DSM-IV criteria and made operational in the NHSDA field study procedures.

Subgroup variations of particular interest include malefemale differences, differences in relation to socioeconomic status, disadvantaged minority status, urban-rural status, and differences in relation to whether the cocaine use history has included crack smoking and injection drug use (eg see Chen and Anthony, 2004). We also take into account the history of early-onset drug use (ie early use of drugs other than cocaine), which might signal a greater susceptibility to become cocaine dependent once cocaine use starts (eg see Anthony and Petronis, 1995).

\section{METHODS}

Data for this study are from public use files of the National Household Surveys on Drug Abuse (NHSDA) for the period 2000-2001, with an aggregate community sample size of 114241 . The NHSDA (recently renamed the National Survey on Drug Use and Health) are ongoing cross-sectional surveys of the drug experiences of US citizens aged 12 years and over. Each year for the past decade, the NHSDA team has drawn nationally representative samples of dwelling units (eg households) and individual respondents, via multistage sampling procedures. Potential participants are allowed to decline or refuse participation in accord with a protocol approved by the cognizant Institutional Review Board. During the 2000-2001 intervals, survey participation was just under $75 \%$.

In this report, we present both weighted and unweighted estimates because the standard NHSDA weighted estimates are based upon more than sample selection probabilities; the weights also incorporate poststratification adjustment for nonresponse under assumptions that the nonrespondents are similar to respondents. It is useful to compare the unweighted and weighted estimates to assess whether and when these assumptions and weighting procedures might be introducing artefacts. A more detailed description of methods and other information on the NHSDA population and each year's sample are available in published reports (SAMHSA, 2001, 2002b).

We combined public use data from the 58680 respondents in CY2000 and 55561 respondents in CY2001 data files in order to assemble a sample of recent-onset cocaine users large enough for this project's analyses. In both years of the survey, the NHSDA assessment protocol to identify recent-onset cocaine users and DSM-IV cocaine dependence cases was the same (SAMHSA, 2002a).

For the 2000-2001 NHSDA assessment of drug experiences, the survey respondents completed a self-administered computerized audio-enhanced interview schedule, and the field interviewer could not see the responses. Each respondent typically must read the questions from the computer screen and listen privately to the audio recording through headphones. A small number of respondents chose to have the interviewer read the questions aloud and then $\log$ the answers for them.

The survey assessment consists of a fixed sequence of standardized preworded and precoded survey questions designed to obtain information about drug experiences and other personal characteristics under study: age, sex, raceethnicity, marital status, education, family income; size of metropolitan statistical area (MSA) is encoded by the survey team. Use of drugs before age 11 years was studied as a possible marker of increased susceptibility to cocaine problems once cocaine use starts. This age limit was chosen to ensure a focus on drug use that started earlier than cocaine use. In this sample, the earliest age of onset of cocaine use was 11 years. The drug categories counted in this susceptibility marker variable were: tobacco, alcohol, cannabis, cocaine, crack, heroin, hallucinogens, inhalants, pain relievers, anxiolytics, stimulants, and sedatives, with categories as defined by the survey team.

For cocaine involvement, each participant was asked a series of standardized questions for 'all forms of cocaine including powder, 'crack,' free base, and coca paste.' These questions elicited information about age of first use, year of first use, and cumulative number of days used in the past 12 months and in the past 30 days.

Recently active cocaine users were asked 11 questions that map onto the seven clinical features of cocaine dependence as per the Diagnostic and Statistical Manual, Fourth Edition (APA, 1994). Analysts on the NHSDA research team used a computerized diagnostic algorithm to identify respondents who endorsed three or more of the seven cocainedependence criteria and coded them as dependent 
(dependence $=1$ ). Recent-onset users who did not meet criteria for dependence, and all others (including those who never used cocaine), were coded ' 0 '.

For this study, we define two kinds of active cocaine users. One group is the 'recent-onset' users, who were identified as individuals who reported using cocaine in any form for the first time within the 24 months prior to assessment. The other group we labeled 'recently-active past-onset' and these are current cocaine users (used in the past year) who began using cocaine more than 2 years before the assessment. The grouping of the recency of onset was ascertained through utilization of an NHSDA-derived variable defining year of first cocaine use. Here, if the difference between the age at assessment and the age first used cocaine was 0 or 1 , the NHSDA team recorded the year of first cocaine use with a range of possible values of 19982000 for the 2000 NHSDA or 1999-2001 for the 2001 NHSDA. Our research team defined those with a nonmissing value for this variable as 'recent-onset'. Respondents for whom the difference exceeded 1 but had used cocaine in the past 12 months were designated by our research team as 'recently-active past-onset'. Owing to the variation in the calendar timing of dates of birth and the dates of the interview, the interval between age at first use and age of interview can be as great as 23 months (just under 24 months). For example, consider a person who used cocaine for the first time late in December 1999 and whose 29th birthday was also in that month, with a CY2001 NHSDA assessment in November of 2001 at age 30 years. The difference between these ages (30-29) has a value of one (1), yet 23 months and some days have elapsed between onset of cocaine use and the NHSDA assessment. Examples of this type help to clarify the concept of 'recent-onset' drug use $v s$ 'past-onset' drug use, which we and others have made operational in a series of prior published scientific articles (eg Anthony and Petronis, 1995; Breslau et al, 2001; Chen and Anthony, 2003, 2004; Storr et al, 2004). It is noteworthy that for some recent-onset users, the elapsed time since first cocaine use is less than 1 month, and for some the elapsed time is as much as 23 months, or just under 24 months. Based upon standard life table and survival analysis conventions, the expected value for mean and median elapsed time, summarized across all recent-onset cocaine users, is about 12 months (ie about midway through the interval from zero months to just under 24 months).

For both weighted and unweighted analyses, STATA 7.0 survey commands were used to conduct logistic regression for complex survey data, with Taylor series linearization to address variance estimation complexities due to nested structures within the multistage sampling plan. The logtransformed odds of cocaine dependence among recent onset cocaine users was regressed upon covariate terms for age, sex, race-ethnicity, route of administration, family income, education, size of MSA, and number of drugs used before age 11 years. Given the study design and focus on occurrence of cocaine dependence among recent-onset cocaine users (a relatively rare outcome), the resulting odds ratios (OR) may be interpreted as relative risk (RR) estimates when the covariates of interest are exogenous, where 'exogenous' means that the covariates are not apt to be responsive to onset of cocaine use or dependence (eg sex, race). Even when the covariates are endogenous (ie responsive to onset of cocaine use or dependence), these OR can be interpreted as a simple gauge of the strength of association between these covariates and the odds of observing cocaine dependence soon after the onset of cocaine use. The $95 \%$ confidence intervals may be used to gauge statistical uncertainty (precision) of the study estimates, and quantitative $p$-values are presented so that readers can gauge strength of the evidence in relation to departures from the null.

\section{RESULTS}

A total of 1081 respondents, $0.95 \%$ of the total sample of 114241 individuals, were found to have started using cocaine for the first time within 24 months of interview assessment. Based on weighted estimates, roughly $0.4 \%$ of the CY2000-2001 NHSDA study population had become a recent-onset cocaine user $(95 \%$ confidence interval, $\mathrm{CI}=0.38, \quad 0.45 \%$, expressed in a range from about 1680000 to 2020000 recent-onset cocaine users during this span of time in the early 21 st century US). Before addressing the risk of becoming dependent, we describe the total sample and the sample subsets in relation to sociodemographic and drug use characteristics.

\section{Sociodemographic Characteristics of Total Sample and Sample Subsets}

Readers interested in the distinction between risk of becoming a cocaine user $v s$ prevalence of persistent cocaine use may wish to compare the 'All Persons' column proportions (weighted and unweighted) to the corresponding proportions in the 'Recently-Active Past-Onset Users' columns and with the 'Recent-Onset Users' columns. The 'All Persons' proportions provide a summary of the characteristics of the unweighted sample and the population under survey (after weighting); in a sense, these are 'expected' values for the population against which we can compare the 'observed' values for cocaine users. For example, in the contrast of weighted proportions, we see that the male-female ratio in the total survey population is 48.1-51.9\%. In the 'Recently-Active Past-Onset Users' column, we see the male-female ratio is $67.1-32.6 \%$ - an observed excess of males relative to the expected population estimate of the male-female ratio. However, in the 'RecentOnset Users' column, we see a relatively smaller male excess: 56.2 vs $43.8 \%$, not appreciably different from the expected values. Here, being male is associated with being a recently-active (persistent) user, with use carried on from past years, as well as with the risk of becoming a recentonset cocaine user.

As compared to the past-onset cocaine users, the recentonset users were slightly younger, as can be seen by comparing either unweighted sample data or weighted population estimates, row by row. For example, looking at weighted values, $35 \%$ of the recent-onset cocaine users were 18-20 years old at the time of survey assessment, as compared to $13 \%$ of the past-onset users, and an estimated $2 \%$ of the recent-onset users were aged 35 years or older, as compared to $37 \%$ of the past-onset users. 
Members of the study population who designate themselves as non-Hispanic White are somewhat over-represented among the recent-onset cocaine users (weighted percent, $\mathrm{WP}=79 \%$ ), and non-Hispanic Black/AfricanAmericans are under-represented among recent-onset cocaine users (WP $=3.9 \%$ vs a value of $10.9 \%$ in the subgroup of past-onset cocaine users and $11.3 \%$ in the study population overall). College seniors or graduates are under-represented among recent-onset cocaine users $(\mathrm{WP}=11 \%)$ as compared to the total population (WP $=22 \%$ ), and those without a high school diploma are over-represented (WP $=40 \%$ among recent-onset cocaine users $v s \mathrm{WP}=26 \%$ for the total study population). Social status differences also are apparent in relation to annual family income, with the recent-onset cocaine users more likely to have values under $\$ 20000$ as compared to the total study population (WP $=30 \%$ for recent-onset cocaine users $v s$ an expected value of $20 \%$ ).

With respect to size of the MSA of residence, there are no remarkable differences in the contrast of recent-onset cocaine users against the total study population. However, past-onset cocaine users are somewhat more likely to live in larger MSAs and somewhat less likely to live in sample segments that are not included within an MSA (eg extremely rural areas of the country).

\section{Dependence and Drug Use Characteristics of Total Sample and Sample Subsets}

Based on weighted estimates, $5.4 \%$ of the recent-onset cocaine users in the study population were found to have become cocaine dependent since onset of cocaine use within 24 months prior to the survey assessment $(95 \% \mathrm{CI}=4,7 \%)$. An estimated $20.5 \%$ of the recently active past-onset cocaine users qualified as recently active cases of cocaine dependence (Table 2). That is, most of the recently-active cases of cocaine dependence in this country had started their cocaine use at some time in the 20th century and do not qualify as recent-onset cocaine users in the 21 st century.

An estimated $2-3 \%$ of the recent-onset cocaine users had used cocaine (all forms) on at least 101 days during the 12 months prior to assessment, as compared to $18.1 \%$ of the recently active past-onset cocaine users. In total, $35 \%$ of the recent-onset cocaine users had consumed cocaine on only 1-2 days in the past 12 months, as compared to $17 \%$ of the recently active past-onset users. The greater accumulated cocaine experience of the past-onset users also can be seen in relation to the history of crack use and injecting cocaine use. For example, an estimated $46 \%$ of the past-onset users had tried crack-cocaine as compared to only $12 \%$ of the recent-onset users, and almost $14 \%$ of the past-onset users had injected cocaine $v s$ an estimated $0.9 \%$ of the recentonset users. With respect to the number of drugs used by age 11 years, the study estimates display no remarkable differences between the past-onset and the recent-onset users (Table 2, last rows).

\section{Risk of Becoming Dependent}

We now focus on the recent-onset cocaine users specifically, seeking answers to the following research question: 'Who is becoming cocaine dependent soon after cocaine use starts?'
That is, whereas this study's estimates indicate that about $5 \%$ of recent-onset cocaine users become cocaine dependent within 24 months of starting cocaine use, this risk of becoming cocaine dependent is not distributed at random among the recent-onset users.

For example, female subjects who start using cocaine are an estimated 3-4 times more likely to become cocaine dependent within 24 months of cocaine onset, as compared to male recent-onset users (estimated unadjusted RR based on weighted data logistic regression analysis, $\mathrm{uRRw}=3.3$; 95\% confidence interval, $\mathrm{CI}=1.7,6.3 ; p<0.001$; Table 3 ). The unweighted RR estimates also are consistent with excess risk of recent-onset cocaine dependence for female subjects, but the point estimates are somewhat smaller than those from weighted analyses. As shown in Table 4, this estimated magnitude of excess risk of becoming cocaine dependent among female cocaine users is statistically independent from associations involving background characteristics (age, race-ethnicity, family income, and number of drugs used by age 11 years): estimated covariate adjusted $\mathrm{RR}$ based on weighted data, $\mathrm{aRRw}=3.2 ; 95 \% \mathrm{CI}=1.6,6.3$; $p=0.001$. In a subsidiary analysis, with the regression model extended to include potentially endogenous covariates that might depend upon levels of the user's cocaine use (level of education, crack-smoking, cocaine-injecting), the parameter estimates for excess risk change very little or not at all $(\mathrm{aRRw}=3.4 ; 95 \% \mathrm{CI}=1.7,6.6 ; p<0.001$; data not shown in Table 4).

With respect to age at assessment, only one age subgroup stands out - namely, the 21-25 year olds who had recently started to use cocaine were somewhat less likely to have become cocaine dependent within 24 months after onset of use, as compared to the reference subgroup of 18-20 year olds (eg Table 3: $\mathrm{uRRw}=0.4 ; 95 \% \mathrm{CI}=0.1,0.9 ; p=0.032$; Table 4 : $a R R w=0.3 ; 95 \% C I=0.1,0.7 ; p=0.008)$. Whereas there may be reasons to think that being an early adolescent signals a markedly excess risk of becoming cocaine dependent soon after starting use, our first RR estimates seem to suggest otherwise. The RR estimates for 12-13 year old and 14-15 year old recent-onset cocaine users are numerically larger than the null value of 1.0 (based on the experience of 18-20 year olds), but the statistical precision of these estimates is not optimal and the associated $p$-values are consistent with fairly weak evidence (eg $p>0.10)$, due in large part to the small number of early-onset users in these early adolescent age subgroups. The RR estimates did not change appreciably when the regression model was extended to include covariate terms for level of education, crack-smoking, and cocaine-injecting (data not shown in Table 4). By implication, risk of becoming cocaine dependent is lower for young people who start at age 21-25 years, but in comparison with these young adult users, there is excess risk of cocaine dependence when the recent-onset user is under the age of 21 years.

Among recent-onset cocaine users, the self-designated non-Hispanic Black/African American users and those in the 'Other' race-ethnicity category are at markedly excess risk of becoming cocaine dependent soon after cocaine use starts. For example, as depicted in the covariate-adjusted RR estimates of Table 4, non-Hispanic Black/African Americans are an estimated nine times more likely to become cocaine dependent within 24 months of starting 
cocaine use as compared to the non-Hispanic White recentonset users $(95 \% \mathrm{CI}=2.9,29.4 ; p<0.001)$. The excess risk for recent-onset cocaine users with African heritage is not attributable to subgroup variation in schooling, crack-smoking, or use of cocaine by injection $(\mathrm{aRRw}=8.7$; $p<0.001$; data not shown in table). The corresponding covariate-adjusted RR estimate for the 'Other' race-ethnicity category is not as extreme, but can be characterized as modest-to-moderate in strength before adjustment for schooling, crack-smoking, and cocaine-injecting $(\mathrm{aRRw}=3.5 ; 95 \% \mathrm{CI}=1.2,10.6 ; p=0.023$; Table 4$)$, and after adjustment for these covariates (aRRw $=4.1 ; 95 \%$ $\mathrm{CI}=1.6,10.5 ; p=0.004$; data not shown in Table 4$)$. The estimated risk of cocaine dependence for Hispanic recentonset cocaine users and for non-Hispanic Whites are not appreciably different $(\mathrm{aRRw}=0.9 ; 95 \% \mathrm{CI}=0.4,2.2$; $p=0.847)$.

Via direct inspection of the race-ethnicity heritage values for the incident cocaine dependence cases in the unweighted sample data on the 'Other' non-Hispanic minority subgroup, we found that a majority of these cases had designated themselves as multiracial (ie as having more than one race-ethnicity heritage). The next most frequent heritage self-designation was non-Hispanic Native American/Alaska Native, followed by non-Hispanic Asian. Finally, the remaining seven cocaine-dependence cases identified themselves as non-Hispanic Native Hawaiian/ Other Pacific Islander.

With social status measured in relation to family income, there was no appreciable variation in risk of becoming cocaine dependent within 24 months of the start of cocaine use (see RR estimates in Tables 3 and 4). Nevertheless, level of schooling or school achievement is an alternative measure of social status, and the study values in Table 1 provided a hint that schooling might be associated inversely with cocaine-dependence risk. In order to probe into this issue, it was necessary to respecify the regression model to produce separate estimates for different age groups, because it is rare for 12-17 year olds to have graduated from high school or to have entered college.

Based upon estimates for respondents old enough to have graduated from high school (age $18+$ years at onset of cocaine use), schooling has an inverse association with risk of becoming cocaine dependent within 24 months after onset of cocaine use. In particular, there is excess risk in the subgroup of recent-onset cocaine users who had not graduated from high school, with lower levels of risk in the subgroup with at least some college. With the older nongraduates as the reference group, the recentonset cocaine users with some college were less likely to have developed cocaine dependence $(\mathrm{uORw}=0.17$; $95 \% \mathrm{CI}=0.05,0.56 ; p=0.004)$; alternately, if we specify users with some college as the reference group, those who had not graduated from high school were an estimated 5-6 times more likely to have become cocaine dependent (ie the inverse of $\mathrm{uORw}=0.17$; data not shown in Table 4). Statistical adjustments for covariates do not yield appreciable attenuation of this odds ratio estimate; however, we must note that level of schooling may qualify as an endogenous covariate with respect to onset of cocaine use. That is, some recent-onset cocaine users in this sample may have dropped out of school or failed to earn their diplomas as a consequence of prior cocaine use.

Just as level of schooling might be endogenous in our analysis, it is also true that the NHSDA-measured values for history of crack-smoking and for history of injection cocaine use might be endogenous. That is, the onset of crack-smoking or injecting cocaine use might start after onset of a cocaine-dependence syndrome, possibly as a consequence of cocaine dependence. Nevertheless, there is reason to study whether cocaine dependence might be observed more frequently among recent-onset cocaine users who have started to smoke crack or who have started to inject cocaine (eg see Chen and Anthony, 2004). As shown in Table 2, roughly $12 \%$ of recent-onset cocaine users have started to smoke crack and about $1 \%$ have started to inject cocaine. The estimates in Table 3 indicate that a noteworthy excess of cocaine dependence is observed among cracksmoking recent-onset users (unadjusted odds ratio based on weighted data, $\mathrm{uORw}=3.4 ; 95 \% \mathrm{CI}=1.7,6.6 ; p<0.001)$ and there is a marked excess of cocaine dependence among cocaine-injecting recent-onset users (uORw $=31.0 ; 95 \%$ $\mathrm{CI}=7.9,120.6 ; p<0.001)$. These estimates do not change appreciably with statistical adjustment for the covariates listed in Table 4 (crack-smoking aORw $=3.7 ; 95 \% \mathrm{CI}=1.8$, 7.4; $p<0.001$; cocaine-injecting aORw $=28.5 ; 95 \% \mathrm{CI}=3.7$, $220.2 ; p=0.001)$, nor with an additional adjustment for level of schooling (data not shown in Table 4).

With respect to size of the Metropolitan Statistical Area of residence at the time of assessment (MSA), estimates based on unweighted NHSDA data indicate that recent-onset cocaine dependence cases are somewhat less likely to be observed in MSAs with 1 million or more inhabitants, as compared to the smaller MSAs (Table 3, unadjusted RR based on unweighted sample data, uORuw $=0.5 ; 95 \%$ $\mathrm{CI}=0.3,1.0 ; p=0.047)$. Nonetheless, NHSDA sample selection probabilities vary by MSA size. Once the NHSDA data are weighted to take these variations into account, the size of the observed occurrence estimates are roughly the same (Table 3: uORw $=0.6$ ), but the association is null-consistent with no variation in risk in relation to MSA size $(95 \% \mathrm{CI}=0.3,1.3 ; p=0.186)$.

Early onset use of alcohol, tobacco, inhalants, or other drugs by age 11 years did not serve as a susceptibility marker for risk of becoming cocaine dependent within 24 months after starting cocaine use. The observed RR estimates are of relatively modest size and the observed $p$-values are consistent with the null hypothesis of no association (ie all $p$-values greater than 0.10 ; Tables 3 and 4 ).

\section{DISCUSSION}

The main findings of this study may be summarized in a few paragraphs. First, recall from this study's introduction the SAMHSA estimate that roughly 1.2 million US citizens started to use cocaine in CY2001. Based on our study estimates, within 24 months after starting cocaine use, an estimated one in 16-20 of these recent-onset cocaine users develop cocaine dependence (ie 5-6\%). Quantified in relation to our $95 \%$ confidence intervals, we estimate that among the 1.2 million recent-onset cocaine users, an 
Table I Selected Sociodemographic Characteristics of all Persons, all Recently-Active Past-Onset Cocaine Users, and the Subset of Recent-Onset Cocaine Users

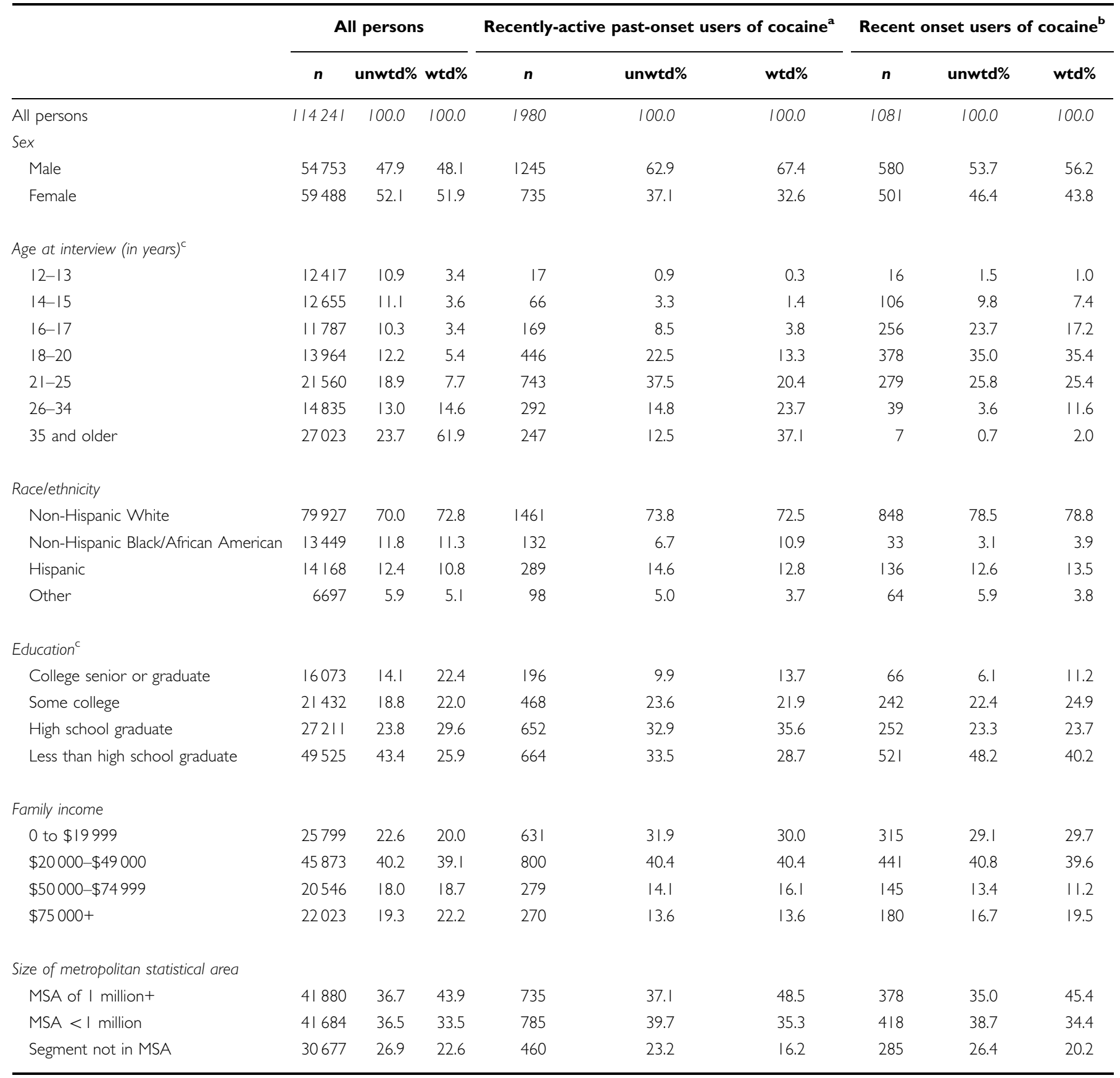

${ }^{a}$ Recently active use in the past 12 months, but with onset 2+ years before survey assessment date.

bithin 24 months of the survey assessment date.

'Reflect values at the time of interview.

Note: 'wtd' indicates weighted data with Taylor series linearization.

Note: values may not sum to $100 \%$ due to rounding error.

Data from 2000-200I National Household Survey on Drug Abuse.

estimated 48 000-84000 have become recent-onset cases of cocaine dependence. Quantified in relation to a US Census estimate of 223280000 US citizens aged 12 years and older, this corresponds to an incidence of about 90-160 newly incident recent-onset cases of cocaine dependence per 100000 persons in the US population.
The risk estimate of 5-6\% developing cocaine dependence soon after onset of use based on the more recently available NHSDA data and a relatively short span of recollection over 24 months is not appreciably different from the NCS risk estimate from the early 1990s (Wagner and Anthony, 2002). However, the NCS estimate of $5-6 \%$ is a predicted risk of 
Table 2 Selected Drug Use Characteristics of all Persons, all Recently-Active Cocaine Users, and the Subset of Recent-Onset Cocaine Users

\begin{tabular}{|c|c|c|c|c|c|c|c|c|c|}
\hline & \multicolumn{3}{|c|}{ All persons } & \multicolumn{3}{|c|}{$\begin{array}{l}\text { Recently-active past-onset } \\
\text { users of cocaine }^{a}\end{array}$} & \multicolumn{3}{|c|}{$\begin{array}{l}\text { Recent onset users } \\
\text { of cocaine }{ }^{b}\end{array}$} \\
\hline & $n$ & unwtd\% & wtd\% & $n$ & unwtd\% & wtd\% & $n$ & unwtd\% & wtd\% \\
\hline All persons & $|1424|$ & 100.0 & 100.0 & 1980 & 100.0 & 100.0 & 1081 & 100.0 & 100.0 \\
\hline \multicolumn{10}{|c|}{ Occurrence of DSM-IV cocaine-dependence syndrome } \\
\hline Yes, $3+$ clinical features & 395 & 0.4 & 0.3 & 329 & 16.6 & 20.5 & 64 & 5.9 & 5.4 \\
\hline \multicolumn{10}{|c|}{ Occasions of cocaine use (all forms) in past 12 months } \\
\hline I-2 days & 744 & 0.7 & 0.4 & 365 & 18.4 & 17.3 & 379 & 35.1 & 35.0 \\
\hline $3-11$ days & 833 & 0.7 & 0.5 & 545 & 27.5 & 25.6 & 286 & 26.5 & 25.5 \\
\hline $12-100$ days & 933 & 0.8 & 0.6 & 752 & 38.0 & 39.0 & 180 & 16.7 & 16.0 \\
\hline \multicolumn{10}{|c|}{ Occasions of crack use in past 12 months } \\
\hline I-2 days & 143 & 0.1 & 0.07 & 93 & 4.7 & 4.2 & 50 & 4.6 & 3.7 \\
\hline $3-11$ days & 126 & 0.1 & 0.08 & 95 & 4.8 & 5.0 & 30 & 2.8 & 2.0 \\
\hline $12-100$ days & 200 & 0.2 & 0.15 & 170 & 8.6 & 10.6 & 30 & 2.8 & 2.2 \\
\hline IOI or more days & $10 \mid$ & 0.1 & 0.08 & 95 & 4.8 & 6.2 & 6 & 0.6 & 0.4 \\
\hline Never/not in past year/dk/ref & $11367 \mid$ & 99.5 & 99.6 & 1527 & 77.1 & 74.0 & 965 & 89.3 & 91.5 \\
\hline \multicolumn{10}{|l|}{ Crack use in lifetime } \\
\hline Yes & 2619 & 2.3 & 2.6 & 843 & 42.6 & 46.0 & 154 & 14.3 & 12.2 \\
\hline No & 111622 & 97.7 & 97.4 & 1137 & 57.4 & 54.0 & 927 & 85.8 & 87.8 \\
\hline । & 10594 & 9.3 & 9.2 & 379 & 19.1 & 16.7 & 165 & 15.3 & 15.8 \\
\hline 2 & 2220 & 1.9 & 1.5 & 148 & 7.5 & 6.9 & 70 & 6.5 & 6.2 \\
\hline 3 & 628 & 0.6 & 0.4 & 99 & 5.0 & 3.6 & 26 & 2.4 & 1.6 \\
\hline
\end{tabular}

a Recently active use in the past 12 months, but with onset $2+$ years before survey assessment date.

bWithin 24 months of the survey assessment date.

Note: 'wtd' indicates weighted data with Taylor series linearization.

Note: values may not sum to 100 due to rounding error.

Data from 2000-200I National Household Survey on Drug Abuse.

observing cocaine dependence onset within 1 person-year after start of cocaine use, and this study's NHSDA estimate is a predicted probability for recent-onset cocaine users, some of whom started to use cocaine as many as 23 months prior to assessment, and some of whom had used cocaine only for a few months. The NHSDA risk estimate is generally consistent with the NCS risk estimate if we apply a standard life-table assumption to the NHSDA data on recent-onset cocaine use, assuming that on average the elapsed person-time from onset of cocaine use to onset of cocaine dependence is roughly 1 year (ie roughly one-half the observed interval from 0 months through 23 months).

The observed risk of becoming cocaine dependent soon after onset of cocaine use was not distributed at random within the population, and the observed variation in risk was not suppressed once study covariates were held constant via regression modeling. Perhaps most intriguing is the excess risk of cocaine dependence observed for female subjects who recently started to use cocaine, in part because Wagner and Anthony (under review) did not find this malefemale difference in their NCS cocaine-dependence risk 
Table 3 Relative Risk Estimates for Becoming Cocaine Dependent among Recent-Onset Cocaine Users, without Statistical Adjustments

\begin{tabular}{|c|c|c|c|c|c|c|c|c|c|}
\hline & \multicolumn{6}{|c|}{ Unweighted $^{\mathrm{a}}$} & \multirow{2}{*}{\multicolumn{3}{|c|}{$\begin{array}{c}\text { Weighted }^{\text {b }} \\
\begin{array}{c}\text { Estimated risk of becoming } \\
\text { dependent }\end{array}\end{array}$}} \\
\hline & \multirow{2}{*}{$\begin{array}{l}\text { Number of } \\
\text { recent onset } \\
\text { cocaine users }\end{array}$} & \multirow{2}{*}{$\begin{array}{l}\text { Number of } \\
\text { cocaine- } \\
\text { dependence } \\
\text { cases }\end{array}$} & \multicolumn{4}{|c|}{$\begin{array}{l}\text { Estimated risk of becoming } \\
\text { dependent }\end{array}$} & & & \\
\hline & & & $\%$ & $\widehat{\mathrm{URR}}$ & $95 \% \mathrm{Cl}$ & $p$-value & $\widehat{\text { uRRw }}$ & $95 \% \mathrm{Cl}$ & $p$-value \\
\hline All persons & 1081 & 64 & 5.9 & - & - & - & 5.4 & - & - \\
\hline Male (ref) & 580 & 21 & 3.6 & 1.0 & - & - & 1.0 & - & - \\
\hline Female & 501 & 43 & 8.6 & 2.5 & $1.5-4.3$ & 0.001 & 3.3 & $1.7-6.3$ & $<0.001$ \\
\hline
\end{tabular}

Age at interview (in years)

$\begin{array}{lr}26+ & 46 \\ 21-25 & 279 \\ 18-20 \text { (ref) } & 378 \\ 16-17 & 256 \\ 14-15 & 106 \\ 12-13 & 16\end{array}$

3
8
25
15
11
2

$\begin{array}{rccllll}6.5 & 1.0 & 0.3-3.4 & 0.981 & 0.6 & 0.1-2.4 & 0.437 \\ 2.9 & 0.4 & 0.2-0.9 & 0.035 & 0.4 & 0.1-0.9 & 0.032 \\ 6.6 & 1.0 & - & - & 1.0 & - & - \\ 5.9 & 0.9 & 0.5-1.7 & 0.702 & 0.7 & 0.3-1.5 & 0.373 \\ 10.4 & 1.6 & 0.8-3.4 & 0.196 & 1.7 & 0.7-4.1 & 0.221 \\ 12.5 & 2.0 & 0.4-9.4 & 0.371 & 4.0 & 0.8-21.5 & 0.101\end{array}$

\section{Race/ethnicity}

Non-Hispanic White (ref)

Non-Hispanic Black

Hispanic

Other

42
5
8
9

5.0
15.2
5.9
$14 . \mid$

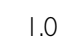

3.4

-

-

1.0

$1.2 \quad 0.6-2.6$

0.647

\section{0}

2.3-20.9

0.001

$\begin{array}{lll}1.2 & 0.6-2.6 & 0.647 \\ 3.1 & 1.4-6.8 & 0.004\end{array}$

0.9

$0.4-2.2$

Education ${ }^{c}$

\section{College senior or graduate}

Some college

High school graduate

graduate (ref)

Family income

$$
\begin{aligned}
& 0 \text { to } \$ 19999 \\
& \$ 20000-49000 \text { (ref) } \\
& \$ 50000-\$ 74999 \\
& \$ 75000+
\end{aligned}
$$

315
441
145
180

19
25
9
11

6.0
5.7
6.2
6.1

1.1
1.0
1.1
1.1

$0.6-2.0$

0.834

4.1

1.6-10.6

0.004

Population density

\section{MSA of I million+}

$M S A<$ I million (ref)

Segment not in MSA

Crack use in lifetime

$$
\begin{aligned}
& \text { No (ref) } \\
& \text { Yes }
\end{aligned}
$$

Ever used needle to inject cocaine

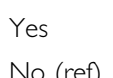

16
1065

1065

No (ref)

$\begin{array}{rccc}3.7 & 0.5 & 0.3-1.0 & 0.047 \\ 6.9 & 1.0 & - & - \\ 10.9 & 1.1 & 0.6-1.9 & 0.827\end{array}$

$0.5-2.4$

0.810

$0.5-2.3$

0.831

1.6

$0.8-3.3$

0.228

1.0

1.2

$0.5-3.3$

$-$

0.697

$0.5-2.6$

0.862

44

20

28.6

2.2

1.0

$\begin{array}{cc}- & - \\ 1.7-5.2 & <0.001\end{array}$
1.0
3.4

1.7-6.6

$<0.00$ ।
0.472

0.003

0.506

- 


\begin{tabular}{|c|c|c|c|c|c|c|c|c|c|}
\hline & \multicolumn{6}{|c|}{ Unweighted $^{\mathrm{a}}$} & \multirow{2}{*}{\multicolumn{3}{|c|}{$\frac{\text { Weighted }^{\mathrm{b}}}{\begin{array}{c}\text { Estimated risk of becoming } \\
\text { dependent }\end{array}}$}} \\
\hline & \multirow{2}{*}{$\begin{array}{l}\text { Number of } \\
\text { recent onset } \\
\text { cocaine users }\end{array}$} & \multirow{2}{*}{$\begin{array}{l}\text { Number of } \\
\text { cocaine- } \\
\text { dependence } \\
\text { cases }\end{array}$} & \multicolumn{4}{|c|}{$\begin{array}{c}\text { Estimated risk of becoming } \\
\text { dependent }\end{array}$} & & & \\
\hline & & & $\%$ & $\widehat{u R R}$ & $95 \% \mathrm{Cl}$ & $p$-value & $\widehat{\mathrm{uRR} w}$ & $95 \% \mathrm{Cl}$ & $p$-value \\
\hline 0 (ref) & 820 & 45 & 5.5 & 1.0 & - & - & 1.0 & - & - \\
\hline I & 165 & 13 & 7.9 & 1.5 & $0.8-2.8$ & 0.236 & 1.6 & $0.7-3.3$ & 0.230 \\
\hline 2 & 70 & 5 & 7.1 & 1.3 & $0.5-3.5$ & 0.565 & 1.5 & $0.4-5.5$ & 0.505 \\
\hline 3 & 26 & I & 3.8 & 0.7 & $0.1-5.2$ & 0.718 & 0.4 & $0.1-3.1$ & 0.386 \\
\hline
\end{tabular}

estimates based upon lifetime retrospection data from the early 1990s. Nonetheless, we note a congruent finding from more recent research based on the NHSDA CY1998 public use data about individual clinical features of the cocaine-dependence syndrome (eg Chen and Anthony, 2004).

Excess risk of becoming cocaine dependent soon after onset of cocaine use also was observed for non-Hispanic Americans of African heritage, and for members of other non-Hispanic race-ethnicity subgroups. Here also, the excess risk was not an artefact of imbalances in histories of crack-smoking or cocaine-injecting, held constant via the statistical modeling.

Recent-onset cocaine users aged 21-25 years were less likely to become cocaine dependent soon after cocaine use, as compared to 18-20 year old recent-onset users. Contrary to what might be expected, very young users (12-15 years old) were not at excess risk of cocaine dependence with the $18-20$ year old users as a reference $(p>0.10)$, but this might be due to the focus on the first 24 months of cocaine use. Anthony and Petronis (1995) found that excess risk of drug problems did not characterize early-onset drug users in the first several years after onset of drug use. The excess risk of drug problems that is associated with early-teen $v$ s late-teen onset of illegal drug use may not be present soon after onset of use, but may require greater elapsed time since first use.

As might be expected based on findings from NHSDA data of prior years, occurrence of cocaine dependence was greater among recent-onset users who had started to smoke crack-cocaine, as compared to those who had not smoked crack (eg see Chen and Kandel, 2002; Chen and Anthony, 2004). In addition, estimated associations between cocaine dependence and cocaine-injecting were very strong, as manifest in odds ratio estimates of 30 or larger, but we are confident that cocaine-injecting is an endogenous covariate (ie responsive to increasing cocaine use), and the $95 \%$ confidence intervals for these estimates are quite wide, due to the relatively small numbers of injecting cocaine users among recent-onset users.

Before more detailed discussion of these study findings on who becomes cocaine dependent soon after onset of cocaine use, and before review of observed null associations, we should acknowledge and discuss several of the more important study limitations. First, we note that the NHSDA study design is cross-sectional, not prospective. Nevertheless, with a focus on recent-onset cocaine users, we have tried to simulate RR estimates that might be observed in a properly conducted prospective study. Perhaps the most prominent threat to validity in this respect is a study design defect in relation to the sampling frame and nonparticipation. That is, based on clinical case reports and ethnographic research, it seems clear that in some individuals the natural history and clinical course of cocaine dependence can be especially aggressive, as manifest in maladaptive behavior or events such as dropping out of school, other disengagements from conventional social bonds, or even drug overdose death, which may occur very soon after onset of cocaine use (eg see Murphy et al, 1989). However, as our research group has discussed elsewhere, it is not clear that the ordinary prospective study design for epidemiological field research can escape these defects and associated threats to validity, once nonparticipation is taken into account. That is, even if the sampling frame for a prospective study sample is $100 \%$ comprehensive in its coverage of individuals with no cocaine experience, we face the companion threat in the form of nonparticipation once cocaine dependence occurs (eg as part of disengagement from social bonds, risk of overdose death). As such, it is not clear that a conventional prospective study design would necessarily yield improvements in these estimates for risk of becoming cocaine dependent soon after onset of cocaine use, which we have derived from the NHSDA crosssectional sampling plan. One potential elaboration of the conventional design involves enlistment of confederates 
Table 4 Relative Risk Estimates for Becoming Cocaine Dependent among Recent-Onset Cocaine Users, with Statistical Adjustment for all Listed Covariates

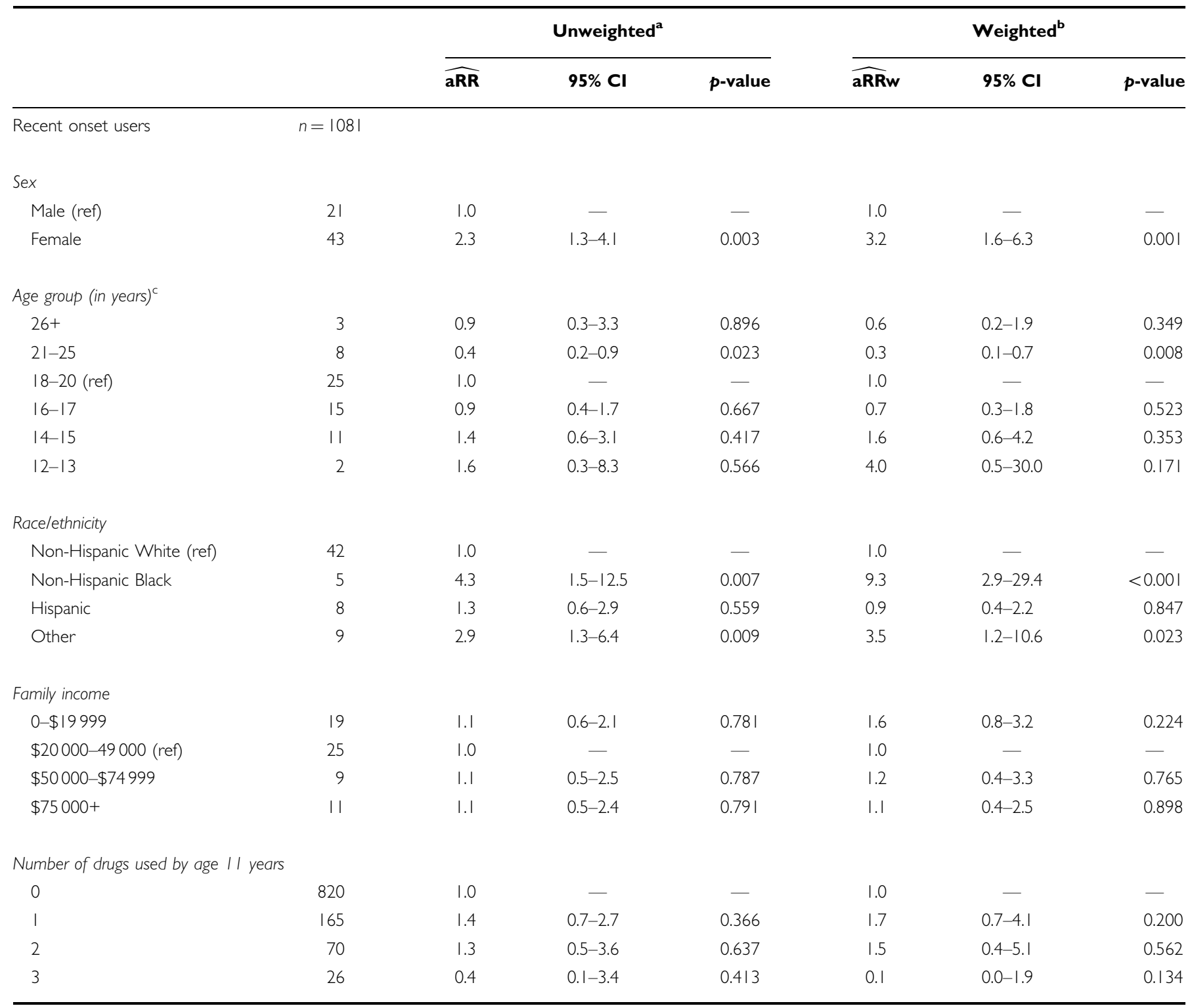

${ }^{a}$ Estimates based on logistic regression, with statistical adjustment for covariates.

bData based on variance estimates via Taylor Series linearization with statistical adjustment for covariates.

'Reflect values at the time of interview.

Data from 2000-200I National Household Survey on Drug Abuse.

who might serve as knowledgeable informants even if the index participant cannot be located, has migrated to the morgue, or declines to sign an informed consent document at the time of follow-up assessment (eg see Anthony et al, 2000; Chen and Anthony, 2004).

Of course, estimates of cocaine problems based on crosssectional samples of the population always must contend with potential differential attrition from the sample, and it has been suggested that epidemiological surveys underestimate the magnitude of truly serious cocaine problems because the most advanced cocaine dependence cases are outside their sampling frames or decline to participate in the surveys (eg see Wagner and Anthony, 2002). There is also concern that cross-sectional research may yield underestimates of rural drug problems, to the extent that rural users may migrate to larger MSAs once the drug dependence process begins. Focus on the most recent onset cocaine users helps to constrain this potential source of error. If advanced cocaine use and cocaine problems make cocaine users less likely to be included in the NHSDA data set, then this source of error may be least prominent when there is a focus on recent-onset cocaine use, except for those users who become disengaged from the household population very rapidly after their first use of cocaine.

Another limitation of note involves the assessment of cocaine use and dependence. In future research, a more 
robust set of estimates might be derived via a combination of cocaine metabolite bioassays, standardized clinical examinations to validate the NHSDA cocaine-dependence assessments, and interviews with knowledgeable informants who can provide valuable information even when a cocaine user agrees to participate but is in a state of denial of cocaine problems at the time of assessment. Even so, the NHSDA computerized self-assessment method might actually be overcoming some of the biases that can emerge in clinical research based on standardized clinical examinations by addiction specialists. For example, if the study aim is to estimate the degree to which crack-smoking or cocaine-injecting might increase risk of cocaine dependence, it might be difficult to avoid a diagnostic work-up bias that would affect the process and results of clinical cross-examination once the clinician-examiner knows that the cocaine user has smoked crack-cocaine or has injected cocaine (eg see Chen and Anthony, 2004).

A third limitation involves the study's specifications for the interval of interest across a span of $0-23$ months after onset of cocaine use, and the cocaine-dependence assessment, which has a focus on the 12 months prior to NHSDA assessment. In future research, it may be possible to align the interval of actual cocaine use with the 12-month interval of cocaine-dependence assessment; NHSDA data in the public use data set do not permit this alignment at present. As a result, our NHSDA risk estimates are based upon an assumption: if a cocaine user has developed the cocainedependence syndrome within the past 24 months, then we assumed that it would be detected by the NHSDA cocainedependence assessment. This assumption might yield a downward bias in this study's risk estimates. However, our research group's prior NCS estimates did not involve this assumption, and as noted above, the NHSDA and NCS risk estimates are not appreciably different.

Other major limitations of the present study hold generally for epidemiological field survey research on illegal drug use and the drug-dependence syndromes. Interested readers will find these limitations discussed in detail elsewhere in reviews and research articles with greater and more specific methodological focus (eg Anthony et al, 1994, 2000; Chen and Kandel, 2002; DeMallie et al, 1995; Grant, 2000; Grant et al, 2003; Gfroerer et al, 2002; Kandel et al, 1997; Kessler et al, 1997; Shaffer and Eber, 2002; Shillington et al, 1995).

Notwithstanding limitations such as these, this new epidemiological field survey evidence on the risk of becoming cocaine dependent soon after onset of cocaine use may deserve attention by cocaine researchers and others who are interested in the public health burdens associated with onset of cocaine use in early 21st century America. Given the estimated expense of treating a single case of cocaine dependence, recently assigned a value between $\$ 1800$ and $\$ 6800$ per episode (Flynn et al, 1999), these estimates for the occurrence of new recent-onset cases of cocaine dependence may help public health officials convey the importance of efforts to prevent cocaine use and to intervene early and soon after cocaine use starts. Even if the lower bound estimates are correct (48000 new cases per year; $\$ 1800$ to treat the first episode), an allocation of $\$ 86400000$ per year would be required to treat this number of new cases. This value does not include the cost of treating past-onset cases of cocaine dependence who have accumulated in the population over the years, and for some of whom prior treatment has not been fully successful.

With respect to risk of becoming cocaine dependent soon after onset of cocaine use, it is noteworthy that excess risk no longer seems to be concentrated within more urban or larger MSAs, although this study's estimates may be distorted by migration toward larger MSAs. Nor does risk of cocaine dependence soon after start of cocaine use show a concentration in the lower social strata of American society, as measured in relation to family income. With respect to the observed female excess in risk of becoming cocaine dependent soon after onset of cocaine use, these results are convergent with some prior research. Studying prevalent cases in the CY1991 to CY1993 NHSDA data, Chen and Kandel (2002) found that cocaine-using female subjects were almost twice as likely as male subjects to be active cases of cocaine dependence (OR $=1.67 ; 95 \%$ $\mathrm{CI}=1.1,2.6)$. Chen and Anthony (2004), studying CY1998 data on recent-onset cocaine users, found that female subjects were more likely than male subjects to have developed clinical features of cocaine dependence soon after onset of cocaine use. These epidemiological findings are consistent with some preclinical data. For example, Hu et al (2004) found neural as well as hormonal differences in rats that may account for sex differences in the regulation of cocaine acquisition.

With respect to the observed quite marked excess risk of cocaine dependence among non-Hispanic recent-onset users of African heritage, we cannot trace the observation to residence in more populous MSAs nor to imbalances in the occurrence of crack-smoking or cocaine-injecting. A similar pattern was seen when Kandel's group studied prevalence of recently active cocaine dependence among all recently active cocaine users (Chen and Kandel, 2002), but that study was more vulnerable to the Neyman prevalent case bias. That is, in the prevalent case data, the observed excess prevalence might be due to a greater duration of cocaine dependence among users of African heritage, more limited access to effective cocaine-dependence treatment, or other consequences of cocaine dependence, whereas the present study's evidence indicates that African heritage is associated with an apparent excess in risk of becoming cocaine dependent soon after onset of cocaine use. This excess risk might be due to greater cocaine availability in areas where non-Hispanic African-American cocaine users live, as opposed to some unusual susceptibility or diathesis for people of African heritage, as noted by Lillie-Blanton et al (1993) in their study of crack-smoking and raceethnicity.

Direct inspection of the self-designated heritage of recentonset cocaine users in our 'Other' minority group has helped to clarify that the observed excess risk of cocaine dependence in this population subgroup might be traced to the experiences of Native American/Alaskan Natives or to those who identify themselves as non-Hispanic with a multiracial background. Nonetheless, in this instance, and in the instance of the excess risk values observed for female subjects and for non-Hispanics of African heritage, more probing research is needed, and this study does little more than point toward future investigations about risk relationships that merit detailed scrutiny. 
The same must be said for the observed inverse association with level of schooling, complicated in part by the above-mentioned endogenous quality of the schooling covariate. In order to clarify this association, we might benefit from more detailed NHSDA data-gathering on the history of schooling experiences and the timing of cocaine use in relation to school achievement. Nonetheless, our most definitive evidence on schooling and illegal drug involvement, including cocaine dependence, will come from careful systems research and randomized preventive intervention trials with the proximal targets being school achievement and the probability of successful graduation from high school or college. If level of schooling is a causal determinant of cocaine use and cocaine dependence, we should see reduced risk of cocaine use and reduced transitions from cocaine use to cocaine dependence among individuals with experimentally induced increased graduation rates.

Several detailed reviews of the issue of crack-smoking and risk of cocaine dependence already have been published (Hatsukami and Fischman, 1996; Chen and Anthony, 2004). This study's evidence is consistent with the idea that risk of cocaine dependence is elevated among crack-smoking cocaine users. Whether it is the crack formulation or route of administration that actually causes the excess risk of cocaine dependence is a question that requires more finegrained detail about the onset of crack-smoking relative to the onset and temporal sequencing of the cocainedependence process. The same is true for our study observation that risk of cocaine dependence is markedly increased among recent-onset cocaine users who inject this drug. Based on preclinical studies and laboratory research with experienced cocaine users, one might infer that cocaine-injecting is a causal determinant of increased risk of cocaine dependence, but definitive field study evidence from outside the laboratory is lacking. Here also, finegrained detail about the onset of cocaine-injecting relative to the cocaine dependence process is required, and in future research it may be possible to use the epidemiological casecrossover design or other procedures that hold constant long-standing personality traits or susceptibility giving rise to (1) onset of cocaine use, (2) onset of crack-smoking, and (3) excess risk of cocaine dependence (eg see Van Ours, 2003; O'Brien et al, under review).

In conclusion, this new field survey research on who becomes cocaine dependent soon after onset of cocaine use has produced some potentially valuable population-level statistics for the US in the early 21 st century. These epidemiological statistics help to clarify the public health burden of recent-onset cocaine dependence in the US and to illuminate the carry-over public health burden associated with cocaine-dependence cases who began using cocaine in the more distant past and who continue to be recently active cases.

We hesitate to assert that these epidemiological estimates have major implications for clinical practice of individual practitioners, except as these estimates might provoke a more aggressive effort to include questions and other assessments for more rapid detection of cocaine use once patients start using this drug. The study implications are more in the realm of public policy and mass action at the level of departments responsible for public health and public safety, especially in relation to prevention of the onset of cocaine use, delaying its onset until the later years of young adulthood when risk of becoming cocaine dependent might be lower, and redoubling of efforts for early detection and intervention as soon as possible after the earliest stages of cocaine involvement.

\section{ACKNOWLEDGEMENTS}

This work was supported by the following NIDA Awards: R01DA09897, K05DA015799, T32DA07202. Data reported herein come from national survey data collected under the auspices of the Office of Applied Studies, Substance Abuse and Mental Health Services Administration.

\section{REFERENCES}

American Psychiatric Association (1987). Diagnostic and Statistical Manual of Mental Disorders, 3rd edn., revised. American Psychiatric Association: Washington, DC.

American Psychiatric Association (1994). Diagnostic and Statistical Manual of Mental Disorders, 4th edn. American Psychiatric Association: Washington, DC.

Anthony JC (1992). Epidemiological research on cocaine use in the USA. Proceedings of the CIBA Foundation Symposium 166, Cocaine: scientific and social dimensions London, England. John Wiley and Sons: Chichester. pp 20-39.

Anthony JC, Neumark YD, Van Etten ML (2000). Do I do what I say? A perspective on self-report methods in drug dependence epidemiology. In: Stone A, Turkan JS, Bachrach CA, Jobe JB, Kurtzman HS (eds). The Science of Self-Report: Implications for Research and Practice. Lawrence Erlbaum Associates: New Jersey.

Anthony JC, Petronis K (1995). Early-onset drug use and risk of later drug problems. Drug Alcohol Depend 40: 9-15.

Anthony JC, Warner LA, Kessler RC (1994). Comparative epidemiology of dependence on tobacco, alcohol, controlled substances, and inhalants: basic findings from the National Comorbidity Survey. Exp Clin Psychopharmacol 2: 244-268.

Behrens DA, Caulkins JP, Tragler G, Haunschmied JL, Feichtinger G (1999). A dynamic model of drug initiation: implications for treatment and drug control. Math Biosci 159: 1-20.

Breslau N, Johnson EO, Hiripi E, Kessler R (2001). Nicotine dependence in the United States: prevalence, trends, and smoking persistence. Arch Gen Psychiatry 58: 810-816.

Chen CY, Anthony JC (2003). Possible age-associated bias in reporting of clinical features of drug dependence: epidemiological evidence on adolescent-onset marijuana use. Addiction 98: 71-82.

Chen CY, Anthony JC (2004). Epidemiological estimate of risk to become dependent upon cocaine: cocaine hydrochloride powder vs crack cocaine. Psychopharmacology 172: 78-86.

Chen K, Kandel D (2002). Relationship between extent of cocaine use and dependence among adolescents and adults in the United States. Drug Alcohol Depend 68: 65-85.

DeMallie DA, Cottler LB, Compton WM (1995). Alcohol abuse and dependence: consistency in reporting of symptoms over ten years. Addiction 90: 615-625.

Flynn PM, Kristiansen PL, Porto JV, Hubbard RL (1999). Costs and benefits of treatment for cocaine addiction in DATOS. Drug Alcohol Depend 57: 167-174.

Gfroerer J, Eyerman J, Chromy J (eds) (2002). Redesigning an Ongoing National Household Survey: Methodological Issues. DHHS Publication No. SMA 03-3768. Substance Abuse and Mental Health Services Administration, Office of Applied Studies: Rockville, MD. 
Grant BF (2000). Theoretical and observed subtypes of DSM-IV alcohol abuse and dependence in a general population sample. Drug Alcohol Depend 60: 287-293.

Grant BF, Dawson DA, Stinson FS, Chou PS, Kay W, Pickering R (2003). The Alcohol Use Disorder and Associated Disabilities Interview Schedule-IV (AUDADIS-IV): reliability of alcohol consumption, tobacco use, family history of depression and psychiatric diagnostic modules in a general population sample. Drug Alcohol Depend 20: 7-16.

Hatsukami DK, Fischman MW (1996). Crack cocaine and cocaine hydrochloride. Are the differences myth or reality? JAMA 276: $1580-1588$.

Hu M, Crombag HS, Robinson TE, Becker JB (2004). Biological basis of sex differences in the propensity to self-administer cocaine. Neuropsychopharmacology 29: 81-85.

Kandel D, Chen K, Warner L, Kessler R, Grant B (1997). Prevalence and demographic correlates of symptoms of last year dependence on alcohol, nicotine, marijuana and cocaine in the US population. Drug Alcohol Depend 44: 11-29.

Kessler RC, Crum RM, Warner LA, Nelson CB, Schulenberg J, Anthony JC (1997). Lifetime co-occurrence of DSM-III-R alcohol abuse and dependence with other psychiatric disorders in the National Comorbidity Survey. Arch Gen Psychiatry 54: 313-321.

Lillie-Blanton M, Anthony JC, Schuster CR (1993). Probing the meaning of racial/ethnic group comparisons in crack cocaine smoking. JAMA 269: 993-997.

Murphy SB, Reinarman C, Waldorf D (1989). An 11-year follow-up of a network of cocaine users. Br J Addict 84: 427-436.

O’Brien MS, Liang KY, Anthony JC (under review). Does cannabis onset trigger cocaine onset?.

Shaffer HJ, Eber GB (2002). Temporal progression of cocaine dependence symptoms in the US National Comorbidity Survey. Addiction 97: 543-554.

Shillington AM, Cottler LB, Mager DE, Compton WM (1995). Selfreport stability for substance use over 10 years: data from the $S t$ Louis Epidemiologic Catchment Study. Drug Alcohol Depend 40: 103-109.
Storr CL, Zhou H, Liang KY, Anthony JC (2004). Empiricallyderived latent classes of tobacco dependence syndromes observed in recent-onset tobacco smokers: epidemiological evidence from a national probability sample survey. Nicotine Tobacco Res 6: 533-545.

Substance Abuse and Mental Health Services Administration (2001). Summary of Findings from the 2000 National Household Survey on Drug Abuse. Office of Applied Studies, NHSDA Series H-13, DHHS Publication No. (SMA) 01-3549. Office of Applied Studies: Rockville, MD.

Substance Abuse and Mental Health Services Administration (2003). Results from the 2002 National Household Survey on Drug Use and Health: National Findings Office of Applied Studies, NHSDA Series H-22, DHHS Publication No. SMA 03-3836. Office of Applied Studies: Rockville, MD.

Substance Abuse and Mental Health Services Administration (2002a). Results from the 2001 National Household Survey on Drug Abuse: Vol. I. Summary of National Findings Office of Applied Studies, NHSDA Series H-17, DHHS Publication No. SMA 02-3758. Office of Applied Studies: Rockville, MD.

Substance Abuse and Mental Health Services Administration (2002b). Results from the 2001 National Household Survey on Drug Abuse: Vol. II. Technical Appendices and Selected Data Tables Office of Applied Studies, NHSDA Series H-18, DHHS Publication No. SMA 02-3759. Office of Applied Studies: Rockville, MD.

Van Ours JC (2003). Is cannabis a stepping-stone for cocaine? $J$ Health Econ 22: 539-554.

Wagner FA, Anthony JC (2002). From first drug use to drug dependence; developmental periods of risk for dependence upon marijuana, cocaine, and alcohol. Neuropsychopharmacology 26: 479-488.

Wagner FA, Anthony JC (under review). Male-female differences in the risk of progression from first use to dependence upon marijuana, cocaine, and alcohol. 